\title{
ANÁLISIS DE LA INCIDENCIA EN EL FLUJO VEHICULAR DE LAS NUEVAS PLATAFORMAS GUBERNAMENTALES EN LA PARROQUIA IÑAQUTO.
}

\author{
ANALYSIS OF THE INCIDENT IN THE TRAFFIC FLOW OF THE NEW \\ GOVERNMENTAL PLATFORMS IN THE PARISH IÑAQUITO.
}

\author{
ANDREA BAQUERIZO ${ }^{1}$; DANIELA CEVALLOS ${ }^{1}$; ARMANDO ECHEVERRÍA ${ }^{1}$; HENRY \\ VILATUÑ $\mathbf{A}^{2}$.
}

${ }^{1}$ UNIVERSIDAD DE LAS FUERZAS ARMADAS - ESPE. Av. general Rumiñahui s/n. Sangolquí, Ecuador.P.Box 171-5-231B, andreabaquerizo22@gmail.com,dscevallos2@espe.edu.ec. henry.vilatunia@ gmail.com.

${ }^{2}$ SECRETARIA DE MOVILIDAD DEL DISTRITO METROPOLITANO DE QUITO. Sucre y Bolivar, Quito, Ecuador, henry.vilatunia@gmail.com.

Recibido: 2 de agosto de 2017 / Aceptado: 23 de enero de 2018

\section{RESUMEN}

La parroquia Iñaquito al ser parte del hipercentro de la ciudad concentra diferentes equipamientos (sociales, culturales, administrativos, etc.) considerados como puntos de atracción; los cuales generan una alta demanda en el flujo vehicular dentro de la zona, ocasionando así que se vuelva imposible mantener una movilidad sostenible. El presente proyecto se enfocó en analizar la incidencia en el aumento del flujo vehicular debido a la implementación de la Plataforma Gubernamental Financiera y del Complejo Judicial Norte definiendo tiempos de viaje con y sin proyectos. La metodología se basó en la determinación de los tiempos de viaje (impedancias); para lo cual se utilizó el promedio semanal del flujo vehicular (abril 2016) en el horario de 7:00 a 8:00 del área de influencia directa de la zona, delimitada por las avenidas: 10 de Agosto, Gaspar de Villarroel, Shyris y Naciones Unidas; con ayuda del software Synchro.8 y tomando en cuenta las diferentes demoras se determinaron las velocidades reales de los vehículos, en un total de 55 intersecciones (232 sentidos) y junto con la distancia de cada una de las avenidas y calles se calcularon los tiempos de viaje 2016; adicionalmente para el cálculo de los tiempos de viaje 2017 se tomó en cuenta: el porcentaje del incremento anual del parque vehicular del DMQ $(5,7 \%)$ y el porcentaje de aumento del flujo vehicular del transporte privado generados por ambos proyectos. Se obtuvo que el $54 \%$ de las vías cercanas a los proyectos presentaron una mayor demanda vehicular lo que ocasionó un aumento en sus tiempos de viaje y que el $46 \%$ mantuvieron igual e incluso disminuyeron sus tiempos de viaje debido a su baja demanda vehicular.

Palabras clave: movilidad, implementación, Iñaquito, impedancia, Synchro.

\begin{abstract}
The parish of Iñaquito, part of the hypercenter of the city, concentrates a variety of features (social, cultural, administrative, etc.) considered points of attraction; these generate a high flow of traffic inside the zone, occasionally to the point at which it becomes impossible to maintain sustainable movement. The present project focuses on analyzing the impact of the increase in the flow of traffic due to the implementation of the Governmental Financial Platform and the Northern Judicial Complex defining trip durations with and without the projects. The methodology is based on determining trip durations; calculated using the average weekly flow of traffic (April 2016) from the hours of 7:00 until 8:00 in the area directly influenced by the zone, demarcated by the following avenues: 10 de Agosto, Gaspar de Villarroel, Shyris, and Naciones
\end{abstract}


Unidas; with the help of Synchro.8 software and taking into account the different delays, the actual speeds of the vehicles are determined, in a total of 55 intersections (232 directions) and together with the distance of each of the avenues and streets, the trip durations 2016 are calculated; additionally, for the calculations of the trip durations 2017 the following were taken into account: the percentage of the annual increase in vehicle parking in the metropolitan district of Quito (5.7\%) and the percentage of increase in the traffic flow of private transportation generated by both projects. It was discovered that $54 \%$ of the roads close to the projects present a large vehicular demand, which caused the increase in the trip durations, and that $46 \%$ stayed the same or decreased their trip durations due to their low vehicular demand.

Key words: mobility, implementation, Iñaquito, impedance, Synchro.

\section{INTRODUCCIÓN}

La parroquia Iñaquito ubicada en el centro norte de Quito, concentra gran afluencia de personas a todas horas del día; actualmente es conocida por ser parte del hipercentro de la ciudad, ya que en ella se encuentran los principales equipamientos que pueden considerarse como una desventaja, ya que convierte a la zona en desarticulada y caótica en cuanto al flujo vehicular. Al añadir: la Plataforma Gubernamental de Gestión Financiera y el Complejo Judicial Norte, los cuales comprenden varios conjuntos de edificios que se definen como núcleos de gestión administrativa, financiera y de servicio público, implicará un crecimiento vertiginoso del parque vehicular, y en la concurrencia de personas, provocando así complejidad en la movilidad vehicular de la zona (Secretaria de Movilidad, 2015).

El décimo primer Objetivo de Desarrollo Sostenible (ODS) fijados en el año 2015 por las Naciones Unidas propone lograr que las ciudades y los asentamientos humanos sean inclusivos, seguros, resilientes y sostenibles (ONU, 2015), así como el Objetivo 7 del plan nacional del buen vivir habla de garantizar los derechos de la naturaleza y promover la sostenibilidad ambiental, territorial y global (PNBV, 2013). Para cumplir con dichos Objetivos se debe contar con estudios sobre gestión en la fluidez vehicular donde se identifique los diferentes modos de transporte que se desplazan a lo largo de la red vial y los aspectos que influyen directamente en el tiempo de viaje que realizan los ciudadanos. (Secretaria de Movilidad, 2015). Es por ello que la presente investigación se planteó la siguiente hipótesis: La implementación de las nuevas plataformas gubernamentales está asociada al incremento de tiempos de viaje en el flujo vehicular dentro de la parroquia Iñaquito.

\section{DESCRIPCIÓN DEL ÁREA DE ESTUDIO}

El proyecto se desarrolló en la zona de influencia directa de la parroquia de Iñaquito, ubicada en la administración zonal Eugenio Espejo del Distrito Metropolitano de Quito, cuya área es de $7,74 \mathrm{Km}^{2}$; lo que corresponde al $6,86 \%$ del suelo urbano de la zona. Los ejes arteriales que delimitan el área de estudio son: Av. Gaspar de Villarroel, Av. 10 de Agosto, Av. Naciones Unidas y Av. De los Shyris; como se muestra en la Figura 1. 


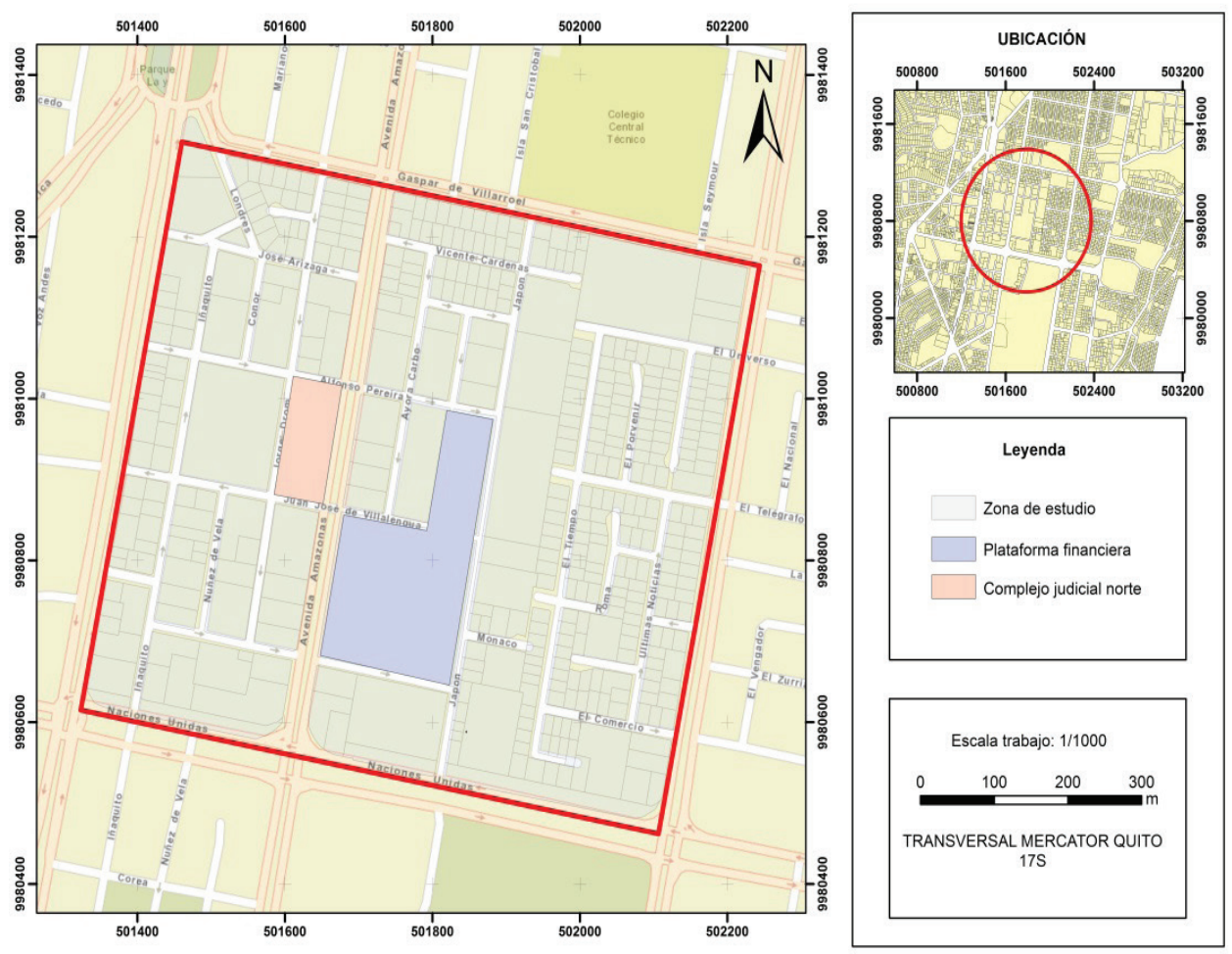

Figura 1: Plano de la zona de influencia directa de la parroquia Iñaquito

\section{METODOLOGÍA}

\section{RECOPILACIÓN Y VALIDACIÓN DE DATOS}

Se recopiló informes técnicos, conteos de flujos vehiculares semanales de todo el mes de abril del año 2016, realizados por la Secretaria de Movilidad, sobre los 58 puntos de muestreo del área de estudio en el horario de 7:00 a 8:00 am.

Los datos primarios del flujo vehicular del mes de abril 2016 fueron levantados, procesados y validados por la Secretaria de Movilidad mediante conteos manuales por técnicos de la institución mientras que los datos secundarios (flujo vehicular semanal abril 2016) fueron validados a través de métodos estadísticos: identificación de los límites de advertencia y de control, a través del cálculo de la media aritmética y desviación estándar, en la Figura 2 se muestra los datos que fueron levantados donde se identifica tanto los movimientos vehiculares como cada una de las direcciones existentes en el área de estudio. 


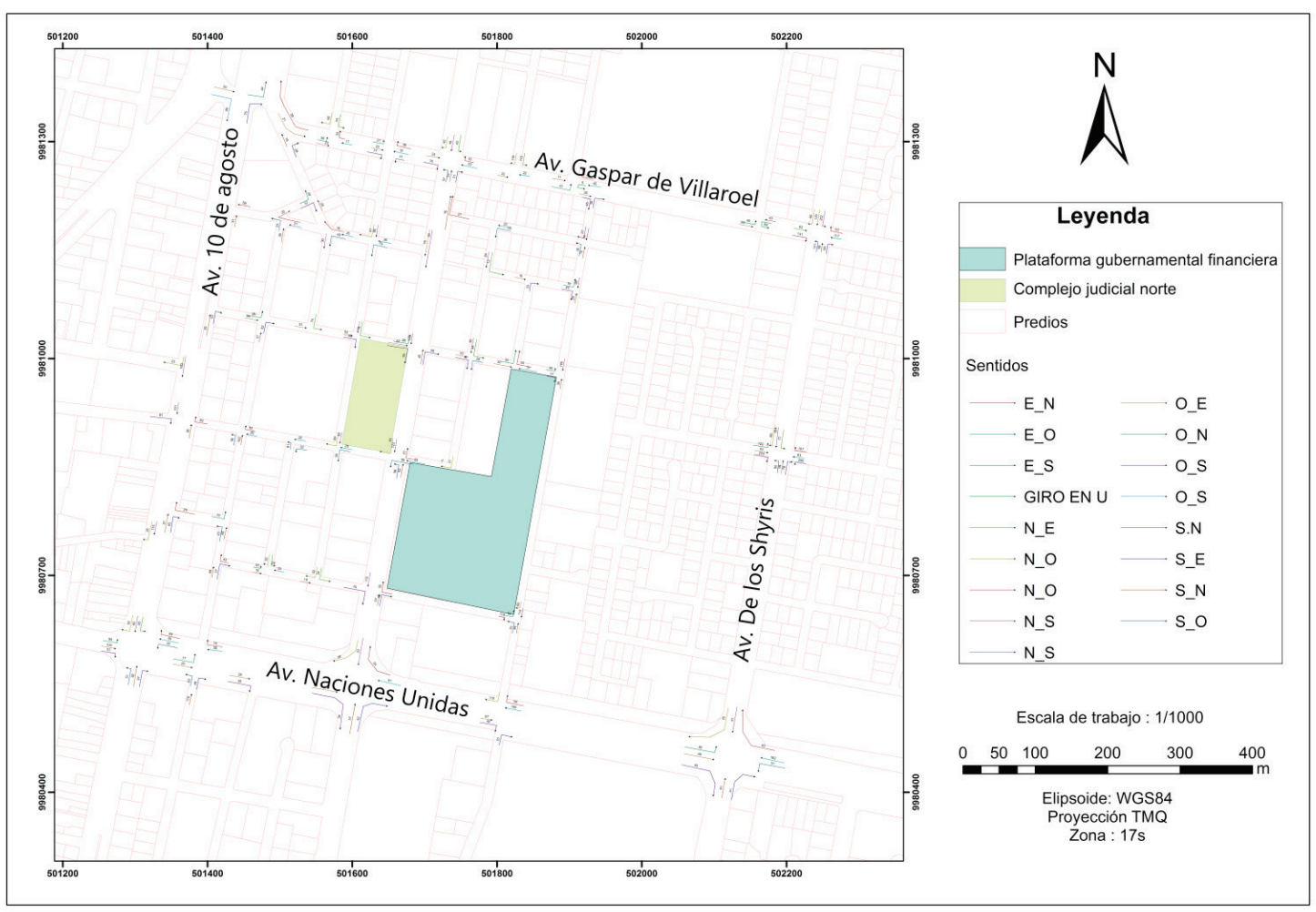

Figura 2. Movimiento y dirección de los datos recolectados

\section{CÁLCULO DE TIEMPOS DE VIAJE 2016}

Dentro del Interfaz del software Synchro 8 se ingresó cartografía base para la construcción de la red vial, se utilizó una restitución georreferenciada del Distrito Metropolitano de Quito del año 2015 escala 1/1000; además se ingresaron valores sobre la configuración vial, flujo vehicular mensual y configuración semafórica para obtener estimaciones de velocidad y distancias de cada una de las intersecciones analizadas.

Para la determinación de la distancia real de cada una de las intersecciones del área de estudio, el software Synchro 8 tomó las distancias trazadas inicialmente con la cartografía base $1 / 1000$, y para la determinación de la velocidad media actual el software elaboró gráficos estadísticos calculadas a través de las demoras de control (d).

\section{- Demoras por control (d)}

La demora por control incluye el movimiento a bajas velocidades y las detenciones en los accesos a las intersecciones, cuando los vehículos disminuyen la velocidad o cambian de posición en la cola (Cal, et al. 2007). Se calcula con la siguiente expresión: 


$$
\mathrm{d}=\mathrm{d}_{1}(\mathrm{PF})+\mathrm{d}_{2}
$$

Donde:

$$
\begin{aligned}
& \mathrm{d}_{1}=\text { demora uniforme }\left(\frac{\mathrm{s}}{\text { vehículos }}\right) ; \mathrm{s}=\text { segundos } \\
& \mathrm{PF}=\text { Factor de ajuste por coordinación } \\
& \mathrm{d}_{2}=\text { demora incremental }\left(\frac{\mathrm{s}}{\text { vehículos }}\right) \\
& \mathrm{d}_{3}=\text { demora por cola inicial }\left(\frac{\mathrm{s}}{\text { vehículos }}\right)
\end{aligned}
$$

- Factor de ajuste por coordinación (PF)

Se denomina a la buena coordinación de semáforos dando como resultado una proporción alta de vehículos que llegan en verde, este factor solo afecta a la demora uniforme (Cal, et al. 2007). Se calcula con la siguiente expresión:

$$
\mathrm{PF}=\frac{(1-\mathrm{P}) \mathrm{f}_{\mathrm{PA}}}{1-\left(\frac{\mathrm{g}}{\mathrm{C}}\right)}
$$

Dónde:

$\mathrm{p}=$ proporción de vehículos que llegan en verde.

$\mathrm{f}_{\mathrm{PA}}=$ Factor de ajuste suplementario por grupos vehiculares que llegan durante el verde. $\mathrm{g} / \mathrm{c}=$ Proporción de tiempo verde disponible.

- $\quad$ Demora uniforme (d1)

La demora uniforme (d1), es la demora que ocurriría si los vehículos llegaran uniformemente distribuidos; tal que no existe saturación durante ningún ciclo (Cal, et al. 2007). Se determina mediante la siguiente expresión:

$$
\mathrm{d}_{1}=\frac{0,5 \mathrm{C}\left(1-\frac{\mathrm{g}}{\mathrm{C}}\right)^{2}}{1-\left[\min (1, \mathrm{Xi}) \frac{\mathrm{g}}{\mathrm{C}}\right]}
$$

Dónde:

$\mathrm{C}=$ duración del ciclo semafórico (s).

$\mathrm{g}=$ tiempo del verde efectivo (s).

$\mathrm{Xi}=$ Relación volumen/ capacidad. 
- Relación volumen/ capacidad

Es el grado de saturación dada por la relación entre capacidad y volumen vehicular para mantener facilidad de transporte. (Wachs et.al., 2000). Se determina mediante la siguiente expresión:

$$
\mathrm{X}_{\mathrm{i}}=\frac{\mathrm{v}_{\mathrm{i}} \mathrm{C}}{\mathrm{s}_{\mathrm{i}} \mathrm{g}_{\mathrm{i}}}
$$

Dónde:

$\mathrm{v}_{\mathrm{i}}=$ Demanda de flujo vehicular actual o proyectado (i) $\left(\frac{\text { vehículos }}{\mathrm{h}}\right)$

$\mathrm{s}_{\mathrm{i}}=$ flujo vehicular de saturacion por carril (i) $\left(\frac{\text { vehículos }}{\mathrm{h}}\right)$

$\mathrm{C}=$ duracion del ciclo semafórico (s)

- Demora incremental (d2)

La demora incremental (d2) toma en consideración las llegadas aleatorias, que ocasiona que algunos ciclos se sobresaturen (Cal, et al. 2007). Se determina mediante la siguiente expresión:

$$
d_{2}=900 T\left[(X-1)+\sqrt{(X-1)^{2}+\frac{8 \mathrm{klX}}{\mathrm{c} \mathrm{T}}}\right]
$$

Dónde:

$\mathrm{T}=$ duración del periodo de analisis $(0,25 \mathrm{~h})$

$\mathrm{k}=$ factor de demora incremental que depende del ajuste de los controles en intersecciones accionadas. $\mathrm{k}=0,5$ para intersecciones predeterminadas.

$1=$ factor de ajuste por entradas de la intersección corriente arriba. $1=1$ para intersecciones predeterminadas.

- Velocidad de viaje (Vv)

Es el promedio de velocidad en $\mathrm{km} / \mathrm{h}$ del flujo de tráfico calculado como la longitud del segmento de la vía dividido para el promedio de los tiempos de las demoras de los vehículos que atraviesan el segmento (Wachs, et.al., 2000). Se determina mediante la siguiente expresión: 


$$
\mathrm{Vv}=\frac{3600 \mathrm{~L}}{\mathrm{Tt}}
$$

Dónde:

$\mathrm{L}=$ longitud del segmento $(\mathrm{km})$

$\mathrm{Tt}=$ tiempo total de demoras $(\mathrm{s})$

Una vez calculados los dos componentes principales para determinar los tiempos de viaje que un vehículo gasta en un determinado segmento vial: distancia recorrida y la velocidad de viaje resultante de las demoras producidas por las intersecciones señalizadas (Wachs, et.al., 2000). Se determina mediante la siguiente expresión:

$$
\mathrm{Vv}=\frac{3600 \mathrm{~L}}{\mathrm{Tt}}
$$

Dónde:

$\mathrm{Tv}=$ tiempo de viaje

$\mathrm{d}=$ distancia recorrida

$\mathrm{v}=$ velocidad de viaje

\section{PREDICCIÓN DE TIEMPO DE VIAJE 2017}

Para el análisis de la generación de tráfico por el flujo vehicular atraído por la plataforma gubernamental financiera y el complejo judicial norte se tomó en:

- Encuestas origen - destino

Para la determinación de la composición del tráfico se basó en los resultados de las encuestas de origen destino, realizadas por la Secretaria de Movilidad 2015 donde se identificó el modo de transporte de los funcionarios que ingresarán a ambos proyectos, teniendo como resultado que ingresaran 1330 vehículos privados y taxis a la Plataforma Gubernamental Financiera (PGF) y 259 vehículos privados al Complejo Judicial Norte (CJN) como se visualiza en la Tabla 1: 
Tabla 1. Modos de transporte de funcionarios a cada uno de los proyectos en estudio.

\begin{tabular}{|c|c|c|}
\hline Modo de transporte & Funcionarios del CJN & Funcionarios de la PGF \\
\hline Transporte público & 253 & 2.470 \\
\hline Transporte particular & 259 & 722 \\
\hline Taxis & - & 608 \\
\hline Otros & 140 & - \\
\hline Total & 652 & 3.800 \\
\hline
\end{tabular}

Para la determinación de la llegada de usuarios al proyecto en el horario de 7:00 a 8:00, se tomó en cuenta de los estudios de impacto ambiental de la plataforma Gubernamental Financiera y el Complejo Judicial Norte, para conocer el número de estacionamientos destinados para usuarios y se consideró la hipótesis que en dicha hora se ocuparía el 25\% de las plazas asignadas, es decir se consideraron 90 vehículos privados de llegada a la plataforma Gubernamental financiera y 49 al Complejo Judicial Norte.

- Distribución espacial del flujo vehicular

Para conocer la distribución espacial de los vehículos privados que ingresaran a los proyectos se tomó en cuenta la Encuesta domiciliaria de movilidad realizada por la Secretaria de Movilidad en el 2011 (EDM, 2011); donde se identificó el porcentaje de ingreso de vehículos privados por cada una de las administraciones zonales del Distrito Metropolitano de Quito a la parroquia Iñaquito, porcentaje que fue asignado al área de influencia directa en estudio como se indica en la Tabla 2:

Tabla 2. Porcentajes de ingreso de vehículos privados por administración zonal

\begin{tabular}{|l|l|l|l|}
\hline \multicolumn{1}{|c|}{$\begin{array}{c}\text { Administraciones } \\
\text { zonales }\end{array}$} & \multicolumn{1}{|c|}{$\begin{array}{c}\text { Vehículos privados } \\
\text { Iñaquito }\end{array}$} & $\begin{array}{c}\text { Vehículos } \\
\text { privados área de } \\
\text { estudio (7-8) }\end{array}$ & $\begin{array}{c}\text { \% vehículos } \\
\text { privados } \\
\text { Iñaquito }\end{array}$ \\
\hline Quitumbe-Sur & 4.438 & 1.081 & $8 \%$ \\
\hline Centro & 2.984 & 727 & $6 \%$ \\
\hline Norte & 24.564 & 5.984 & $46 \%$ \\
\hline La delicia- Calderón & 16.320 & 3.976 & $30 \%$ \\
\hline Tumbaco & 3.570 & 870 & $7 \%$ \\
\hline Los Chillos & 2.095 & 510 & $4 \%$ \\
\hline
\end{tabular}

- Planteamiento de rutas de acceso

Existen varias posibilidades en cuanto a rutas de acceso a los proyectos, sin embargo la presente investigación estableció un escenario tomando en cuenta una de dichas posibilidades. A continuación se indica como ejemplo las rutas de acceso establecidas 
para la administración zonal Quitumbe - Sur urbano hacia los proyectos tomando en cuenta el porcentaje de ingreso de vehículos privados calculados anteriormente y expuesto en la Tabla 2.

Quitumbe - Sur Urbano (8\%):

» Desde la Av. Naciones Unidas (redondel), Iñaquito, Alfonso Pereira y Japón

» Desde la Av. Naciones Unidas (redondel), Iñaquito y Alfonso Pereira.

Para el cálculo de los tiempos de viaje 2017 se utilizó la misma metodología que para el cálculo de tiempos de viaje 2016 realizando cambios en la red vial en el software Synchro 8 , el ingreso del nuevo flujo vehicular tomando en cuenta el aumento de vehículos privados por cada uno de los proyectos y el aumento del parque automotor anual $(5,7 \%)$, para así generar nuevas velocidades y poder calcular los nuevos tiempos de viaje con la presencia de los proyectos.

\section{VALIDACIÓN DE TIEMPOS DE VIAJE 2017 / HIPOTESIS}

- Validación de tiempos de viaje 2017

La Validación de datos se la realizó a través de la verificación de tiempos de viaje en el área de estudio. Se tomó una muestra de población finita del total de intersecciones al 95\% de confiabilidad. La selección de las intersecciones se la realizó a través del criterio del muestreo por conglomerados (población en grupos parecidos) y para seleccionar las intersecciones se utilizó el criterio del muestro sistemático. Una vez realizada la verificación de los tiempos de viaje en salidas de campo se validaron a través de pruebas de hipótesis para comprobar la semejanza de los datos cálculos y los datos medidos en campo. A continuación se muestra las pruebas de hipótesis que se realizaron.

Prueba F para varianzas de dos muestras

$$
\mathrm{F}_{\text {prueba }}=\frac{\mathrm{s}_{\mathrm{x}}^{2}}{\mathrm{~s}_{\mathrm{w}}^{2}}
$$

Dónde:

$\mathrm{S}_{\mathrm{x}}^{2}=$ Estimación de varianzal

$\mathrm{S}_{\mathrm{w}}^{2}=$ Estimación varianza2

Prueba T (Varianzas poblacionales desconocidas pero supuestamente iguales) 


$$
\begin{gathered}
t=\frac{(\bar{x}-\bar{y})-\left(\mu_{x}-\mu_{1}\right.}{s \sqrt{\frac{1}{n_{X}}+\frac{1}{n_{Y}}}} \\
S=\sqrt{\frac{\left(n_{X}-1\right) S_{x}^{2}+\left(n_{Y}-1\right) s_{y}^{2}}{n_{X}+n_{Y}-2}}
\end{gathered}
$$

Dónde:

$\overline{\mathrm{x}}, \overline{\mathrm{y}}=$ promedios variable 1,2

$\mu_{\mathrm{x}}, \mu_{\mathrm{y}}=$ porcentaje de las variables

$\mathrm{S}_{\mathrm{x}}^{2}, \mathrm{~S}_{\mathrm{y}}^{2}=$ varianza muestral 1,2

$\mathrm{n}_{\mathrm{x}}, \mathrm{n}_{\mathrm{y}}=$ número de datos variable 1,2

- Validación de la hipótesis (tiempos abril 2016 vs tiempos abril 2017)

Una vez calculados los tiempos de viaje abril 2016 - abril 2017 se realizó la prueba Z de distribución de diferencias de medias muestrales para dos poblaciones normales.

$$
\mathrm{z}=\frac{(\overline{\mathrm{x}}-\overline{\mathrm{y}})-\left(\mu_{\mathrm{x}}-\mu_{\mathrm{y}}\right)}{\sqrt{\frac{\sigma_{\mathrm{X}}^{2}}{\mathrm{n}_{\mathrm{X}}}+\frac{\sigma_{\mathrm{y}}^{2}}{n_{Y}}}}
$$

Dónde:

$\overline{\mathrm{x}}-\overline{\mathrm{y}}=$ promedios variable 1,2

$\mu_{\mathrm{x}}-\mu_{\mathrm{y}}=$ porcentaje de las variables

$\sigma_{\mathrm{x}}^{2}-\sigma_{\mathrm{y}}^{2}=$ desviación de la variable 1,2

$\mathrm{n}_{\mathrm{X}}-\mathrm{n}_{\mathrm{Y}}=$ número de datos variable 1,2

\section{RESULTADOS}

Una vez comparados los tiempos de viaje 2016 - 2017 se obtuvo que de un total de 232 sentidos; en 125 se presentó un aumento de tiempos de viaje en un rango de 0,04 a 3,88 min; en 77 sentidos se mantuvieron sus tiempos y en los 30 restantes se verificó una disminución de tiempos de viaje en un rango de 0,07 a 1,19 min; como se muestra en la Tabla 3. 
Tabla 3: Comparación de los tiempos de viaje 2016 - 2017

\begin{tabular}{|l|l|l|}
\hline Número de sentidos & Comparación de tiempos de viaje 2016 - 2017 & Rangos de tiempo \\
\hline 125 & Aumentó & $0,04-3,88 \mathrm{~min}$ \\
\hline 77 & Igual & $0 \mathrm{~min}$ \\
\hline 30 & Disminuyó & $0,07-1,19 \mathrm{~min}$ \\
\hline
\end{tabular}

A continuación se muestra la distribución del conjunto de datos correspondientes a los resultados de los tiempos de viaje Abril 2016 y 2017; como se observa en la figura 3 y 4.

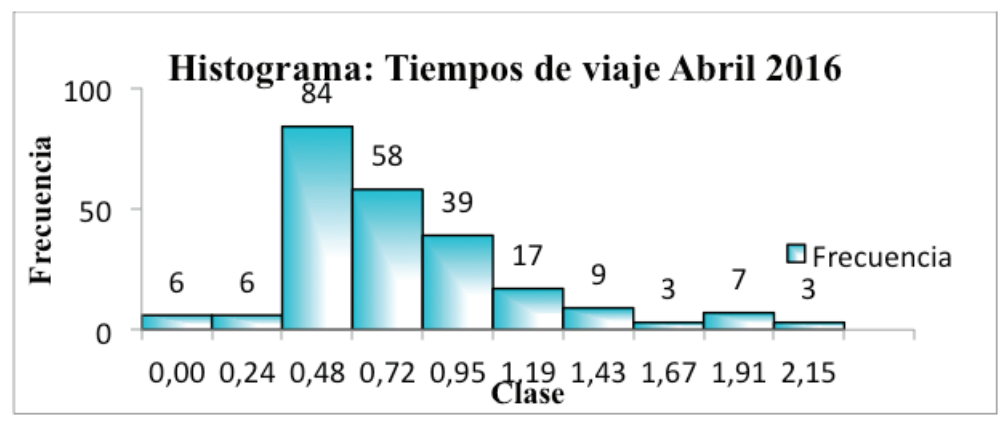

Figura 3. Histograma de la distribución de los tiempos de viaje Abril 2016.

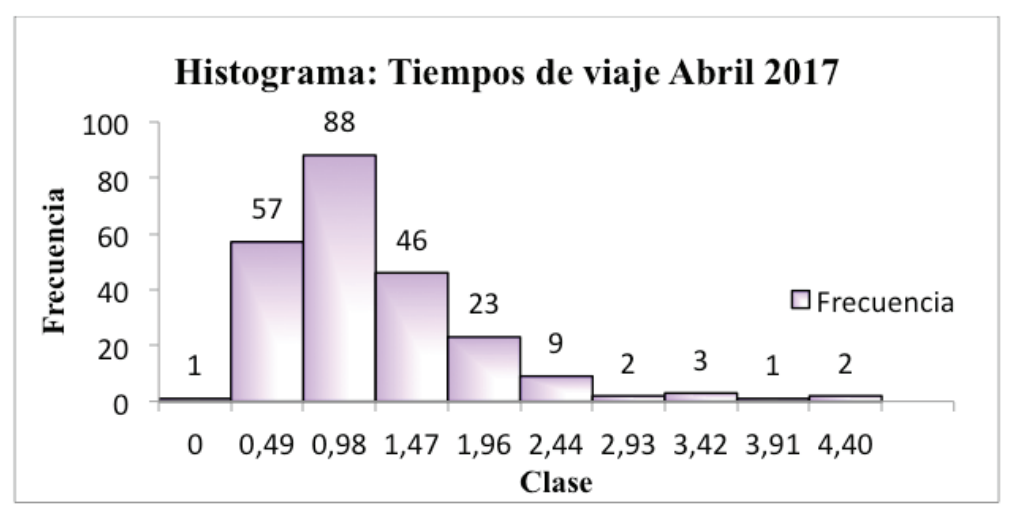

Figura 4. Histograma de la distribución de los tiempos de viaje Abril 2017.

En porcentaje, dichos resultados representan: el 54\% a los 125 sentidos que aumentaron sus tiempos de viaje, el 33\% a los 77 sentidos que mantuvieron sus tiempos y el 13\% a los 30 sentidos que disminuyeron sus tiempos de viaje. Lo anterior expuesto se muestra a continuación en la Figuras 5 y 6, donde se muestra el porcentaje de aumento / disminución en los tiempos de viaje y los resultados del software Synchro 8 donde se verifica la disminución de velocidades en el área de estudio. 


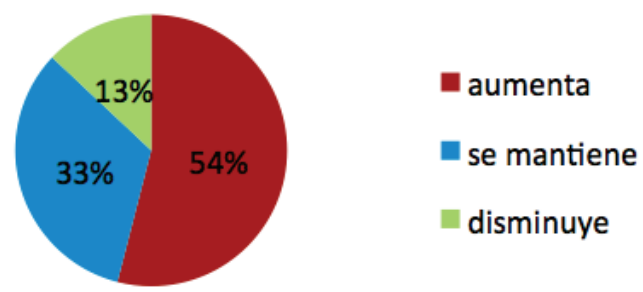

Figura 5. Análisis de tiempos de viaje 2016 - 2017

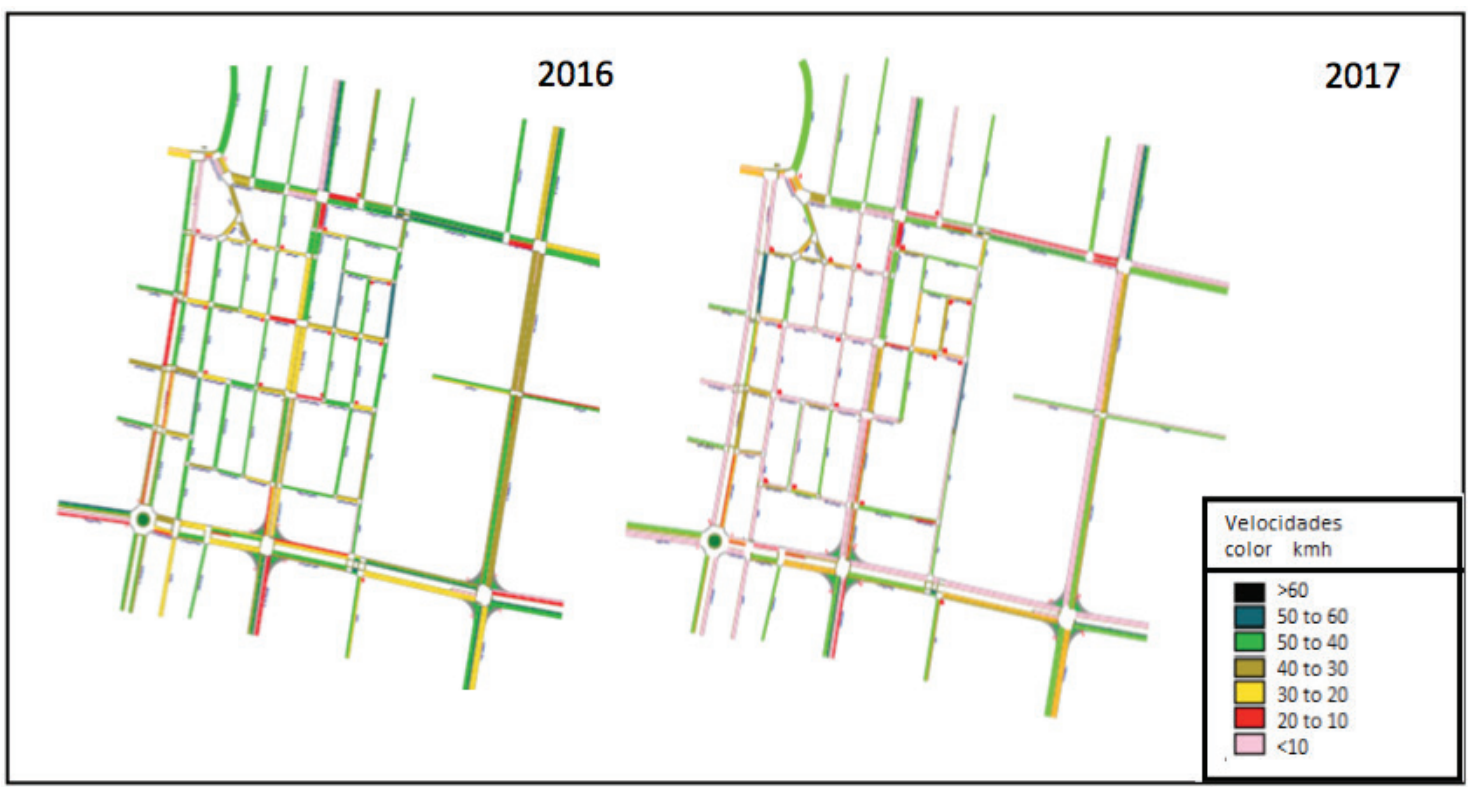

Figura 6. Resultados de la disminución de velocidades 2016-2017 en el software Synchro 8

De esta manera se lograron establecer las vías de afectación por la incidencia de ambos proyectos; se determinó que algunas de las vías con una afectación alta fueron: la Av. Naciones Unidas, Av. Amazonas, Av. Gaspar de Villarroel, Av. De los Shyris; entre otras. Como resultado de este análisis se obtuvo un plano a escala de trabajo 1:1000; el cual representó la afectación de la incidencia de los proyectos a las diferentes direcciones de todas las vías del área de estudio, como se muestra en la Figura 7.

Para la comprobación de la hipótesis se realizó la prueba $\mathrm{Z}$ para varianzas poblacionales conocidas en donde la hipótesis nula fue rechazada (U1 $\geq \mathrm{U} 2$ ); donde U1 representa los tiempos de viaje 2016 y U2 los tiempos de viaje 2017 por lo tanto se aceptó la hipótesis alternativa (U1 < U2) lo que significó que los tiempos de viaje abril 2017 fueron mayores a los tiempos de viaje abril 2016. 


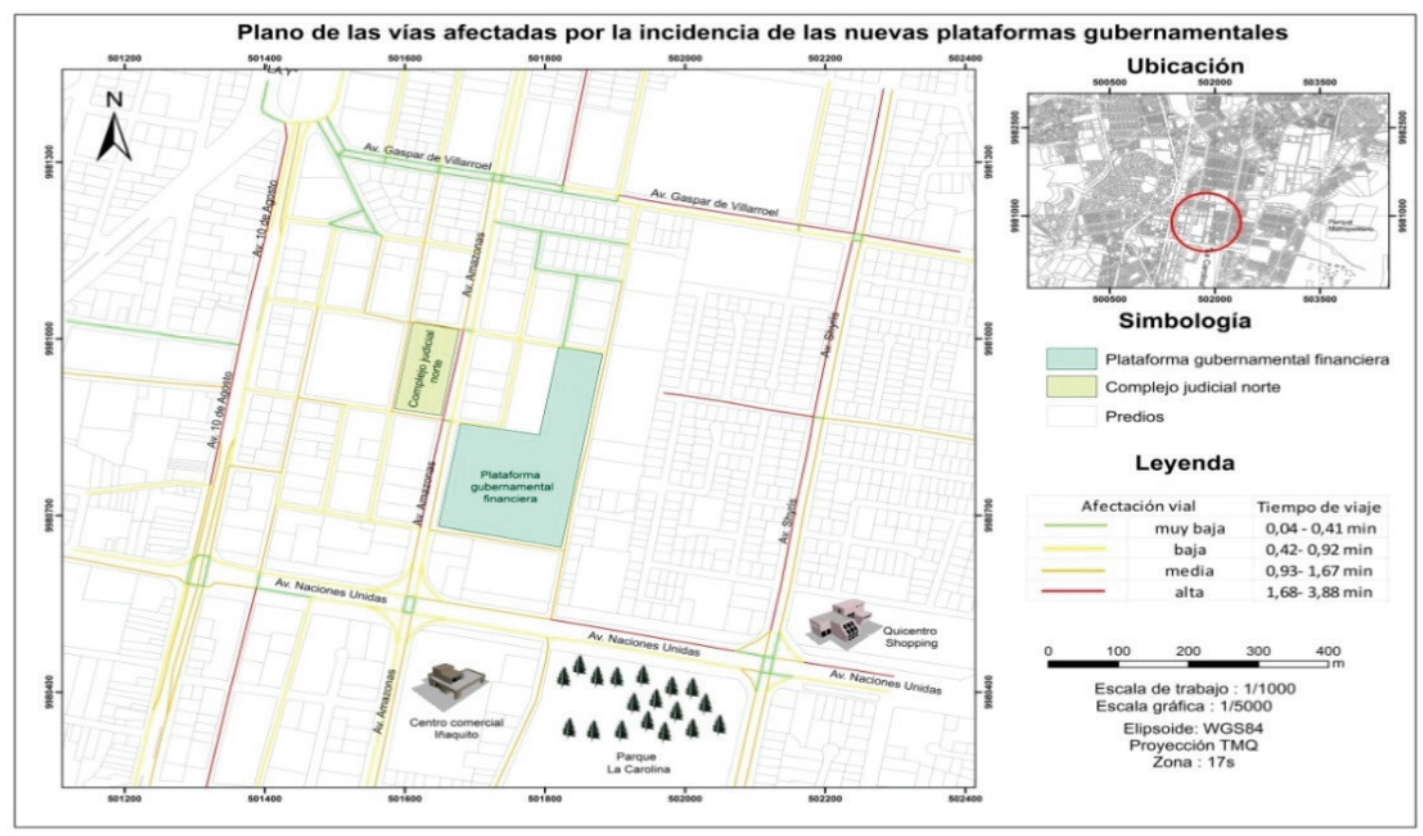

Figura 7. Plano de las vías afectadas por la incidencia de los proyectos

\section{DISCUSIÓN}

Los 125 sentidos con un aumento en sus tiempos de viaje representaron el 54\% del total; dicho porcentaje se evidenció en las vías de acceso a los nuevos proyectos, debido a que los conductores tienen una mayor preferencia hacer uso de estas vías para sus diferentes viajes a lo largo del día. El 46\% representado por aquellos sentidos cuyos tiempos de viaje se mantuvieron o incluso disminuyeron se hizo evidente en vías con una menor demanda vehicular y en vías que no están vinculadas con el acceso a los proyectos.

En dicho análisis se tomaron en cuenta ciertas variables vinculadas al congestionamiento vehicular como por ejemplo: características físicas de las vías, presencia de señales de tránsito, duración de los ciclos semafóricos, entre otras; sin embargo para un análisis más complejo seria indispensable considerar otras variables relacionadas, como: la presencia de peatones, presencia de bicicletas o motocicletas, presencia de las paradas formales del transporte público e incluso tomar en cuenta otros equipamientos como centros de recreación, escolares u otras instituciones públicas que se encuentren dentro de la zona. Es importante también mencionar que el horario escogido para el desarrollo del proyecto fue de 7:00 a 8:00 horas, debido a que se consideró el horario de entrada de los funcionarios a los proyectos; sin embargo la zona de estudio al ser generadora de una gran cantidad de viajes cuenta con diferentes horarios a lo largo del día que reflejan una alta demanda vehicular; por lo tanto en futuros estudios sería importante analizar el flujo vehicular dentro de la zona tomando en cuenta diferentes horarios. 


\section{CONCLUSIONES}

Dentro del área de estudio existió un aumento de tiempo de viaje en 125 sentidos en un rango de 0,01 a 3,07 minutos, lo que represento un 54\%; en 77 sentidos se mantuvieron los mismos tiempos de viaje que represento el 33\% y en 30 sentidos hubo una disminución en sus tiempos de viaje en un rango 0,07 a 1,19 lo que represento un $13 \%$.

Las rutas óptimas generadas para cada uno de los proyectos gubernamentales en la zona de estudio fueron 7 con diferentes orígenes: norte, centro-sur, valles; de las cuales en el escenario 2016 se obtuvo un rango en sus tiempos de viaje de 3-11 minutos de viaje y en el escenario 2017 se mantuvieron en su mayoría las mismas rutas con un aumento en sus tiempos de viaje de 0-6 minutos, a excepción de las entradas centro-sur y valles que generaron rutas diferentes debido a que las opciones anteriores generaban mayores tiempos de viaje.

La hipótesis que se planteó en la investigación: La implementación de las nuevas plataformas gubernamentales está asociada al incremento de tiempos de viaje en el flujo vehicular dentro de la parroquia Iñaquito; fue validada mediante pruebas de hipótesis y aceptada como verdadera.

\section{AGRADECIMIENTOS}

Los autores agradecen a la Secretaria de Movilidad por la colaboración en el desarrollo y en la obtención de la información requerida para la investigación realizada.

\section{REFERENCIAS}

Cal., R, et al. , (2007). Ingeniería de tránsito. México D.F., México: Alfaomega grupo editor.

Organización de las Naciones Unidas (O.N.U.) (2015). Objetivos de desarrollo sostenible. http:// www.un.org/sustainabledevelopment/es/cities/. Ingresado: 23 de agosto del 2016.

Plan Nacional del Buen Vivir (PNBV), (2013). Objetivos del plan nacional del buen vivir. http:// www.buenvivir.gob.ec. Ingresado el 23 de agosto del 2016.

Secretaría de Movilidad (2011). Encuesta domiciliaria de Movilidad 2011.Quito, Ecuador.

Secretaría de Movilidad (2015). Diagnóstico de la movilidad en el distrito metropolitano de quito para el plan metropolitano de desarrollo territorial (PMOT). http://gobiernoabierto.quito. gob.ec. Ingresado: 23 de agosto del 2016.

Wachs, M. et.al. (2000). High capacity manual (HCM). Washington, Estados Unidos. 